\title{
Utilização de uma boneca terapêutica como estratégia educativa para cuidados e prevenção do pé diabético
}

\author{
Use of a therapeutic doll as an educational strategy for diabetic foot care and prevention \\ Uso de muñeca terapéutica como estrategia educativa para el cuidado y prevención del pie \\ diabético
}

Recebido: 17/03/2021 | Revisado: 23/03/2021 | Aceito: 29/03/2021 | Publicado: 08/04/2021

\author{
Thaís Valdeci da Rocha Ferro \\ ORCID: https://orcid.org/0000-0001-5401-174X \\ Faculdade CESMAC do Sertão, Brasil \\ E-mail: thaisrferro@gmail.com \\ Núbia Ivo da Silva \\ ORCID: https://orcid.org/0000-0002-7857-9185 \\ Faculdade CESMAC do Sertão, Brasil \\ E-mail: nubbiaivo@gmail.com \\ Myllena Tavares Bezerra \\ ORCID: https://orcid.org/0000-0002-9257-1737 \\ Faculdade CESMAC do Sertão, Brasil \\ E-mail: myllenabezerra@outlook.com \\ João Paulo do Nascimento Cordeiro \\ ORCID: https://orcid.org/0000-0003-3407-1328 \\ Faculdade CESMAC do Sertão, Brasil \\ E-mail: joaopaulodnc@gmail.com \\ Renata da Silva Miranda \\ ORCID: https://orcid.org/0000-0002-05523-1954 \\ Universidade Estadual do Ceará, Brasil \\ Faculdade CESMAC Do Sertão, Brasil \\ E-mail: renamirandaaa@gmail.com \\ Daniele Cristina de Oliveira Lima da Silva \\ ORCID: https://orcid.org/0000-0003-4248-697X \\ Docente da Faculdade CESMAC do Sertão, Brasil \\ E-mail: daniele.silva@cesmac.edu.br
}

\begin{abstract}
Resumo
Esta pesquisa tem por objetivo demonstrar a relevância da utilização da boneca terapêutica como estratégia educativa para cuidados e prevenção do pé diabético. Trata-se de uma pesquisa-ação qualitativa de natureza observacional, com analise dos artigos publicados nas bases de dados disponíveis na BVS: BDENF; LILACS e SciELO. Desenvolvemos as oficinas proporcionando a sensibilização e conscientização da população sobre prevenção e cuidados com pé diabético e com muita satisfação conseguimos difundir os resultados para a comunidade, entre outros acadêmicos e até mesmo profissionais que conheciam essa abordagem de metodologia ativa de ensino. Por fim, fazemos coro afirmando que a realização de atividades lúdicas com idosos é um importante aliado para o fortalecimento da autonomia destes indivíduos. Ressalta-se a importância do desenvolvimento de atividades grupais pelos enfermeiros voltadas para os idosos na atenção primária, principalmente para a prevenção de incapacidades e complicações relacionadas às doenças crônicas, em particular, o DM.
\end{abstract}

Palavras-chave: Diabetes Mellitus; Pé diabético; Autocuidado; Brinquedo; Educação em enfermagem.

\begin{abstract}
This research aims to demonstrate the relevance of using the therapeutic doll as an educational strategy for the care and prevention of diabetic foot. This is a qualitative action research of an observational nature, with analysis of the articles published in the databases available in the VHL: BDENF; LILACS and SciELO. We developed the workshops providing awareness and awareness of the population about prevention and care with diabetic foot and with great satisfaction we were able to disseminate the results to the community, among other academics and even professionals who knew this approach of active teaching methodology. Finally, we chorus stating that the performance of recreational activities with the elderly is an important ally for strengthening the autonomy of these individuals. It is emphasized the importance of the development of group activities by nurses aimed at the elderly in primary care, mainly for the prevention of disabilities and complications related to chronic diseases, in particular, DM.
\end{abstract}


Keywords: Diabetes Mellitus; Diabetic foot; Self-care; Toy; Nursing education.

\section{Resumen}

Esta investigación tiene como objetivo demostrar la relevancia del uso del muñeco terapéutico como estrategia educativa para el cuidado y prevención del pie diabético. Se trata de una investigación acción cualitativa de carácter observacional, con análisis de los artículos publicados en las bases de datos disponibles en la BVS: BDENF; LILACS y SciELO. Desarrollamos los talleres dando a conocer y concienciando a la población sobre la prevención y atención del pie diabético y con gran satisfacción pudimos difundir los resultados a la comunidad, entre otros académicos e incluso profesionales que conocían este enfoque de metodología de enseñanza activa. Finalmente, coro afirmando que la realización de actividades recreativas con las personas mayores es un aliado importante para fortalecer la autonomía de estos individuos. Se destaca la importancia del desarrollo de actividades grupales por parte de enfermeras dirigidas al adulto mayor en atención primaria, principalmente para la prevención de discapacidades y complicaciones relacionadas con enfermedades crónicas, en particular, la DM.

Palabras clave: Diabetes Mellitus; Pie diabético; Autocuidado; Juguete; Educación en enfermería.

\section{Introdução}

O Diabetes Mellitus (DM) é uma doença crônica não contagiosa que provoca mudanças na vida das pessoas, necessita de cuidados peculiares para o adequado manejo da doença, requer colaboração entre as pessoas com DM, familiares e profissionais de saúde. Seu aparecimento pode estar relacionado a fatores hereditários ou ambientais. Alvo constante atenção à dieta, exercícios físicos, monitoramento da glicose e, em muitos casos, uso de medicação (Boell, et al., 2014).

A hiperglicemia permanente que caracteriza o diabetes mellitus (DM) traz clinicamente uma carga de doença aos seus portadores pelas complicações crônicas e pelo aumento na mortalidade nesses pacientes. O pé diabético é o episódio final das complicações crônicas do DM e os principais fatores fisiopatológicos da ulceração e infecções nos membros inferiores compreendem a neuropatia diabética, a pressão plantar e o trauma. Outros elementos contribuintes são a doença arterial periférica (DAP) de graus variados e distúrbios no processo de cicatrização e na defesa imunológica (Scain, et al., 2018).

Gomes (2017) reitera que no pé diabético dá-se o comprometimento do sistema nervoso periférico sensitivo, motor e autonômico, com redução da sensibilidade do pé, com consequente deformidades, atrofia da musculatura interóssea, aumento do arco plantar, dedos em "garra" e calos em áreas de aumento de pressão, colaborando para o aparecimento de bolhas, calos, lesões e úlceras. Desta forma, há maior risco para amputações não traumáticas.

O pé diabético é um importante problema de saúde pública, pois é o principal fundamento de internação e de gastos hospitalares de pacientes com diabetes mellitus. A taxa de mortalidade em pacientes diabéticos é alta, com uma taxa de 5 milhões de mortes decorrentes da diabetes, registrado em 2015. Pacientes diabéticos com úlceras nos pés apresentam uma taxa de mortalidade duas vezes maior quando contraposto com a de pacientes diabéticos sem úlceras nos pés (Cardoso, 2018).

O Brasil é o quarto país com o maior número de pessoas com DM no mundo. Estima-se que, atualmente, 14,3 milhões de brasileiros tenham a doença. Esse quadro exige medidas urgentes para controlar e prevenir o DM, bem como suas complicações. Caso contrário, o país deve ter um número significativo de pessoas com sequelas graves e com expectativa de vida reduzida pela alta taxa de mortalidade causada pela doença (Lucoveis, 2018).

A diligência da Doença Periférica (DP) está relacionada à orientação profissional em práticas simples, como o conhecimento da sensibilidade dos pés e suas implicações, higiene adequada e hidratação dos pés, seleção de calçados apropriados e autoexame dos pés. Ainda assim, sabe-se que o grau de escolaridade interfere na amplificação dessas habilidades e no acesso às informações relevantes para o autocuidado (Rodrigues, 2014).

Para identificar possíveis alterações vasculares é importante inspecionar a pele, que pode ser atrófica e brilhante, com cabelos diminuídos ou ausentes, extremidades frias, unhas grossas e envoltas. Os MI (membros inferiores) pode apresentar palidez na elevação e declividade na descarga. A aparição de claudicação intermitente ou dor em repouso, pulsos tibiais e pés 
diminuídos ou ausentes à palpação sugerem Doença Arterial Periférica (DAP). A suspeita de DAP pode ser verificada pelo cálculo do Índice Tornozelo-Braquial (ITB) (Lucoveis, 2018).

O conhecimento dos principais determinantes da DM é imprescindível para compor políticas e projetos voltados para diminuir a ocorrência desse problema de saúde pública na população, principalmente em indivíduos da comunidade, cenário de atuação oportuna, para propor ações preventivas para controlar esses determinantes que podem contribuir desfavoravelmente no estado de saúde (Roediger, 2018).

Para tanto, o espaço acadêmico deve entusiasmar a construção do conhecimento necessário à formação do enfermeiro generalista, baseado no rigor científico, intelectual e ético. As Diretrizes Curriculares Nacionais de 2001 (DCN) para cursos de graduação em enfermagem afirmam que o enfermeiro deve ter conhecimento e atuar sobre os problemas de saúde mais prevalentes no perfil epidemiológico nacional, com ênfase na região de ação da epidemiologia e nos fatores biopsicossociais determinantes (Silva, 2018).

Entende-se que, com ações conjuntas entre os serviços de saúde, profissionais que nele atuam, indivíduos e famílias, é possível o crescimento de estratégias para identificar precocemente fatores de risco para o pé diabético, e assim evitá-los e/ou controlá-los (Cecilio, 2015).

A educação voltada para a autogestão é imprescindível para o paciente com diabetes, a qual deve favorecer a aquisição de conhecimento, habilidades e instruí-lo para o autocuidado, a fim de melhorar os resultados clínicos, estado de saúde e a qualidade de vida. Os estudos de intervenção correlacionadas ao autocuidado em diabetes estabelecem uma relação positiva com as ações de promoção da saúde e manutenção de ações de autocuidado na população de adultos (Macedo, 2017).

Para tamanho, o enfermeiro deve fazer uso de diversos recursos educacionais nos diferentes cenários da prática profissional para intervir no processo de ensino e aprendizagem à pessoa com pé em risco, destacando-se o uso de tecnologias educativas, como recursos lúdicos para educação em saúde, a fim de melhorar o exercício do autocuidado em indivíduos com DM (Galdino, 2019).

A extensão universitária é um processo educativo, científico e cultural que busca a articulação entre ensino e pesquisa, é compreendida como uma constituição de saberes compartilhados, em via de mão dupla, entre acadêmicos e a comunidade. Tem-se como consequência a produção de conhecimento, por meio da vivência com a realidade brasileira e regional. Assim, a Universidade retorna para a comunidade o aprendizado que foi submetido à reflexão teórica (Freitas, 2016).

O desenvolvimento deste trabalho justificou-se devido à relevância da criação de uma boneca terapêutica como ferramenta para educação em saúde de pacientes diabéticos, abordando o tema pé diabético de forma lúdica, potencializando as orientações a serem realizadas e a compreensão do que é dito.

Desse modo, este estudo tem como objetivo relatar a experiência vivenciada por meio de uma ação extensionista sobre uma boneca terapêutica que promove a sensibilização e conscientização da população sobre o pé diabético. Demonstrar a relevância da utilização da boneca terapêutica como estratégia educativa para cuidados e prevenção do pé diabético.

\section{Metodologia}

\subsection{Tipo de estudo}

Trata-se de uma pesquisa-ação, de natureza básica e possui uma abordagem qualitativa. Este tipo de pesquisa preocupase com o universo de significados, motivos, aspirações, atitudes, entre outros, estabelecendo as ações e relações entre as pessoas (Minayo, 2012).

A metodologia usada baseou-se nos fundamentos de pesquisa-ação que considera de acordo com Grittem, et al., (2008) algumas questões comuns relativas ao método, tais como a participação dos envolvidos na pesquisa, o papel da reflexão sobre a 
realidade a ser estudada, a necessidade de administração do conhecimento e a ética do processo. A escolha deste caminho metodológico relaciona-se ao fato de aproximar-se da realidade social, visando, assim, o conhecimento científico, sem desconsiderar os significados e crenças dos envolvidos nos processos da vida cotidiana que passam por transformações internamente ligadas ao modo de viver dos idosos diabéticos, assinalando a importância de trabalharmos com as complexidades, as especificidades e as diferenciações internas dos objetos que, precisam ser contextualizados e tratados na sua singularidade.

Corroborando esta ideia, consideramos que a pesquisa-ação favorece o desenvolvimento de um processo de interação entre pesquisadores e os sujeitos participantes da pesquisa. Acreditamos que ela apresenta, como potencialidade, aproximar os pesquisadores com os trabalhadores, profissionais do serviço de saúde e a comunidade, bem como diálogo entre a ciência e a vida (Pessoa, 2013).

\subsection{Fase exploratória}

É de suma importância a assistência de enfermagem na orientação dos pacientes com diabetes. Se torna indispensável, uma vez que prevenção é uma das melhores maneiras para evitar as complicações do diabetes, é importante também que o paciente queira aprender a respeito, para que haja prevenção correta do pé diabético. E daí surge a necessidade de um profissional capacitado para auxiliar e orientar os pacientes nesta etapa de aprendizado sobre o autocuidado.

\subsection{Formulação do problema}

Com a falta de atenção que os diabéticos têm com os pés, por serem cuidados simples acabam sendo dispensados na rotina do seu dia-a-dia. A falta de conhecimento da população diabética sobre a prevenção e os cuidados adequados ocasiona o surgimento de lesões nos pés. Sendo assim, por meio de ações educativas, serão orientados pacientes diabéticos, familiares e cuidadores sobre como prevenir e cuidar do pé diabético.

\subsection{Construção de hipóteses}

Devido à falta de conhecimento dos pacientes diabéticos de como cuidar corretamente dos pés, surge a necessidade da realização de ações educativas que irão sensibilizar sobre a importância dos cuidados e prevenção do pé diabético. Espera-se com essas ações de sensibilização, minimizar consideravelmente o surgimento de lesões nos pés dos pacientes diabéticos, diminuindo a demanda de curativos na atenção primária, melhorando a qualidade de vida destes pacientes.

\subsection{Realização de seminário}

Desenvolveu-se ações educativas na atenção primária com os idosos diabéticos, familiares e cuidadores, que proporcionaram a sensibilização e conscientização da população sobre a prevenção e cuidados com pé diabético, havendo construção coletiva de conhecimentos por meio das ações realizadas, nas quais foram trocados saberes com os pacientes diabéticos.

Atualmente, pensa-se ser possível afirmar, de forma sintética, que a observação participante se caracteriza pela promoção de interatividade entre o pesquisador, os sujeitos observados e o contexto no qual eles vivem.

A pesquisa dita qualitativa e dentre todas as suas técnicas, em particular, a observação participante, obriga o pesquisador a lidar com o "outro", num verdadeiro exercício constante de respeito à autoridade, pressupõe convívio intercâmbio de experiências primordialmente através dos sentidos humanos: olhar, falar, sentir, vivenciar e experimentar (Augusto, 2013).

Utilizamos a boneca terapêutica idosa Aurora com instrumento facilitador para o entendimento da neuropatia diabética, que trouxe como exemplo um pé bem cuidado e saudável e o outro, cujos cuidados foram negligenciados, mostrando a origem das lesões. 


\subsection{Seleção de amostra}

Idosos diabéticos, seus familiares e respectivos cuidadores.

\subsection{Coleta de dados}

Observamos os diabéticos, seus familiares e cuidadores nas oficinas realizadas, podendo assim examinar o conhecimento e as dúvidas que eles apresentavam sobre o aparecimento da lesão neuropática diabética, a prevenção e os cuidados.

\subsection{Análise e interpretação dos dados}

Do ponto de vista de organização e análise dos dados, fez-se a opção pela Teoria Fundamentada nos Dados. Esta escolha metodológica teve como principal meta possibilitar a apreensão de uma realidade concreta a partir da dinâmica cotidiana das interações humanas, por meio das atividades da ação extensionista. Este tipo de opção surgiu devido à hipótese de que "extrair" da prática cotidiana as formas de interação entre os idosos e os facilitadores das ações educativas, analisando como os aspectos apresentados durante as oficinas poderiam oferecer novos elementos sobre a maneira pela qual, na prática, podemos pensar em alternativas de acolhimento e escuta, subsidiando ações de educação continuada.

Neste sentido, a opção pelo referencial metodológico da Teoria Fundamentada nos Dados (Grounded Theory-GT) mostrou-se pertinente, pois tem em seu fundamento a proposta de construir teorias a partir do trabalho empírico ou de um privilégio do trabalho de campo (Charmaz, 2009).

A principal hipótese que baseia a GT é: “A geração da teoria vinda dos dados significa que mais hipóteses e conceitos não somente vêm de dados, mas são sistematicamente trabalhados a partir da relação com os dados durante o curso da pesquisa" (Glazer \& Strauss, 1967).

Pode-se considerar que a teoria é gerada através de três etapas: coleta de dados, codificação e análise. Tais etapas dãose conjuntamente, pois, para os autores da GT, separá-las impediria a geração da teoria.

\subsection{Plano de ação}

Temos por objetivo primordial fazer com que a população tome conhecimento sobre a importância da prevenção e dos cuidados que devem ter como o pé diabético. Serão beneficiados de forma que os pacientes, os familiares e os cuidadores terão uma postura correta para conseguir minimizar este erro que ocorre corriqueiramente.

Outro ponto importante a ser trabalhado é que as ações educativas buscam uma forma de levar informação para essa população de forma clara e objetiva, para que esses pacientes se conscientizem da importância de mudar os hábitos de vida. Então, assim, verão mudanças significativas em seu dia-a-dia.

Outra postura a ser adotada na atenção primária de saúde é implantar oficinas educativas que devem ser comandadas pela Enfermagem. A atenção primária representa a porta de entrada dos pacientes para obter assistência, para que haja a conscientização e a sensibilização do cuidado com o pé diabético, levando à população a opção de serem implantados os cuidados em seu cotidiano, com eficácia e facilidade.

\subsection{Divulgação dos resultados}

Após a realização das oficinas, foram elaborados periódicos para serem levados aos eventos, nos quais foi apresentado, pela primeira vez, o trabalho intitulado "Boneca Terapêutica: estratégia educativa para cuidados e prevenção do pé diabético em atenção primária à saúde", no VIII Congresso Cendovascular Internacional, em Arapiraca-AL. O trabalho foi premiado em primeiro lugar. O segundo periódico foi apresentado no Simpósio Internacional de Enfermagem- SIE, da Faculdade CESMAC 
do Sertão em Palmeira dos Índios-AL. Foi intitulado por "Cuidados e prevenção do pé diabético - relato de ações extensionistas" e foi Premiado em segundo lugar.

\subsection{Fontes das publicações}

Foram usadas as bases de dados da Biblioteca Virtual de Saúde (BVS): Scientific Electronic Library Online (SciELO), literatura científica e técnica da América Latina e Caribe (LILACS), Base de dados em Enfermagem (BDENF) e Google Acadêmico.

Como estratégias de busca, foram utilizados os Descritores em Ciências da Saúde (DeCS): "Diabetes Mellitus"; "Pé Diabético"; "Autocuidado"; "Brinquedo"; "Educação em Enfermagem"; nos idiomas inglês e português. Os descritores foram utilizados primeiro, de forma isolada e depois, com a combinação do booleano "AND".

\subsection{Critérios de inclusão}

- Publicados em português; publicados no período entre 2010 e 2019; disponíveis na íntegra.

\section{Critérios de exclusão}

Artigos publicados protegidos para download e artigos que não estivessem disponíveis na íntegra gratuitamente.

\subsection{Aspectos éticos}

Por se tratar de uma pesquisa-ação qualitativa de natureza observacional, e, consequentemente, não utilizar humanos como objeto de pesquisa, de acordo com a Resolução número 466, de 12 De dezembro de 2012 do Conselho Nacional de Saúde (CNS - ministério da Saúde - MS), não houve a necessidade de que esta produção se submetesse ao CEP (Comitê de Ética e Pesquisa).

\section{Resultados}

A seguir, serão apresentados os resultados da experiência de problematização sobre metodologias de aprendizagem ativas.

\subsection{Construção das oficinas educativas}

Nesta, o grupo responsável pela execução do projeto, constituído por uma docente de enfermagem da Faculdade Cesmac do Sertão - Campus Palmeira dos Índios, buscou entrar em contato com a literatura para ter embasamento sobre o tema: utilização de uma boneca terapêutica como estratégia educativa em ações extensionistas para cuidados e prevenção do pé diabético.

Mediante a busca realizada observamos que não havia boneco terapêutico para pacientes adultos e diabéticos, frente a esse achado surgiu o interesse dos discentes acerca da temática.

Ressalta-se também a escassez do uso de boneco terapêutico com adultos e idosos. Todos os componentes do grupo entraram em discussão sobre o tema, para que houvesse a promoção da integração dos estudantes e a construção coletiva do problema a ser tratado, havendo, assim, a participação ativa de todos os alunos no processo de ensino-aprendizagem.

Houve uma reflexão coletiva entre todos os componentes do grupo, na qual a pauta era as indagações que seriam abordadas, tais como: a falta de conhecimento da população diabética sobre os cuidados adequados com os pés e as complicações que podem surgir no decorrer do tempo.

Abordando o assunto de uma forma mais crítica para que sejamos compreendidos de forma clara e objetiva, elaboramos pontos essenciais a serem estudados sobre o tema, como: a falta de cuidado que os diabéticos têm com os pés (por serem simples 
eles dispensam essa pratica do seu dia-a-dia); a falta de conhecimento da população diabética sobre a prevenção e os cuidados adequados para que não surjam lesões.

Nessa etapa, o aluno é protagonista por buscar a melhor forma de conhecimento, possibilitando confrontar os diferentes modos que há de ensinar e aprender o tema descrito. Sendo assim, criamos um grupo no WhatsApp para viabilizar a comunicação, no qual resolvemos como iria se dá a criação da boneca.

A princípio seria usado um pé de manequim de plástico, porém, ao discutirmos, percebemos que teria uma boa aderência para construir a lesão nele, então uma artesã construiu a boneca idosa de feltro e em seguida outra artesã fez as lesões. Dessa forma, houve a construção coletiva de conhecimentos e a troca de saberes por meio das oficinas realizadas, possibilitando o compartilhamento do poder entre os indivíduos envolvidos no processo de ensino-aprendizagem.

Produzimos a boneca terapêutica, que foi a proposta do grupo, juntamente com a docente responsável. Conseguimos marcar por nossa inovação e criatividade, tanto pelo fato de não existir boneca terapêutica para o tratamento com adultos e também por ser uma metodologia ativa de ensino.

Isso refletiu de forma que todos ficaram engajados para desenvolver as oficinas, fazendo uso de uma metodologia ativa que priorizou o ensino-aprendizagem, de forma que todos saíram ganhando.

Apresentamos a boneca idosa terapêutica de nossa criação e mais uma vez surpreendeu a todos pela sua inovação. Mediante a isso, nós, acadêmicos, adotamos um comportamento de protagonismo no nosso aprendizado acerca do tema proposto pela docente, já que buscamos artigos e fizemos a leitura de várias temáticas correlacionadas, a fim de atingirmos a população de forma inovadora e eficaz para a prevenção e tratamento do pé diabético.

Nossa proposta de aplicação à realidade foi elaborada coletivamente, já que todos compartilhávamos de ideias parecidas. Com o decorrer do tempo, elas foram moldadas para chegarmos em um consenso: atingir a população em geral, que poderia compartilhar os saberes com os demais, mas, principalmente, com os pacientes diabéticos. Então, realizamos oficinas na Estratégia Saúde da Família (ESF), onde foi possível haver essa captação precoce, por ser a porta de entrada na comunidade na assistência de saúde.

O primeiro encontro foi realizado para falar sobre a boneca terapêutica, no qual socializamos todo o conhecimento que fora produzido com a criação da boneca. Utilizamos as metodologias ativas na implantação das oficinas, nas quais os pacientes diabéticos se aprofundaram mais em como prevenir e cuidar do pé diabético. Isso foi possível graças a prática lúdica de terapia para adultos com boneco que não existia até então.

Desenvolvemos as oficinas, proporcionando a sensibilização e conscientização da população sobre a prevenção e cuidados com pé diabético e com muita satisfação, conseguimos difundir os resultados para a comunidade, para outros acadêmicos e até mesmo para profissionais que já conheciam essa abordagem de metodologias ativas de ensino.

\section{Aplicação das oficinas}

Facilitadores: Thaís Ferro; Carla Eduarda; Myllena Tavares; Núbia Ivo e João Paulo.

Data: $16 / 03 / 2019$

\section{Apresentação:}

Realizamos nossa primeira oficina educativa para falar sobre a boneca terapêutica, na qual socializamos com os idosos diabéticos, familiares e seus respectivos cuidadores todo o conhecimento que fora produzido com a criação da boneca. Utilizamos as metodologias ativas na implantação das oficinas, nas quais os pacientes obtiveram mais conhecimento de como prevenir e cuidar do pé diabético, usando como inovação a lúdica terapia para adultos com boneco que não existia até então.

Local da atividade: Dia 16 de abril em Palmeira dos Índios - AL na Unidade Básica de Saúde São Francisco.

Público alvo: Idosos diabéticos, seus familiares e respectivos cuidadores; presentes quatorze pessoas. 
Objetivo geral: Proporcionar à comunidade informações de como prevenir e cuidar do pé diabético, voltadas a realidade de cada um e adequando os devidos cuidados de forma correta.

\section{Objetivos específicos da atividade:}

- Apresentar a oficina de forma simples e direta na Unidade Básica de Saúde.

- Sensibilizar a população da importância em prevenir e cuidar do pé diabético corretamente.

Competências a serem alcançadas: reconhecer a saúde como direito e condições dignas de vida e atuar de forma a garantir a integralidade da assistência, entendida como conjunto articulado e contínuo das ações e serviços preventivos e curativos, individuais e coletivos, exigidos para cada caso em todos os níveis de complexidade do sistema.

Facilitadores: Thaís Ferro; Carla Eduarda e João Paulo.

Data: $10 / 05 / 2019$

\section{Apresentação:}

Oficina apresentada para os indivíduos diabéticos e não diabéticos ensinando os devidos cuidados que devem ter com os pés e quais as principais consequências do diabetes não tratado, demonstrando os materiais ideias para a execução do cuidado e os fatores que atrapalham e geram danos ao indivíduo.

Local da atividade: Dia 10 de maio em Arapiraca - AL no Centro de Referência Integrado de Arapiraca no ambulatório de feridas.

Público alvo: Indivíduos diabéticos e não diabéticos, seus familiares e respectivos cuidadores, presente quinze pessoas.

Objetivo geral: Levar informações relevantes, que conscientizem a população sobre o autocuidado, prevenção e cuidados com o pé diabético.

\section{Objetivos específicos da atividade:}

- Apresentar a oficina de forma simples e direta na Unidade Básica de Saúde.

- Sensibilizar a população da importância em prevenir e cuidar do pé diabético corretamente.

Competências a serem alcançadas: reconhecer a saúde como direito e condições dignas de vida e atuar de forma a garantir a integralidade da assistência, entendida como conjunto articulado e contínuo das ações e serviços preventivos e curativos, individuais e coletivos, exigidos para cada caso em todos os níveis de complexidade do sistema.

Facilitadores: Thaís Ferro e Núbia Ivo.

Data: 09/07/2019

Apresentação:

Oficina realizada na UBS Santa Terezinha com o tema "Prevenção do pé diabético", junto com a equipe do NASF, abordando a prevenção de quedas, que também são fatores relevantes para o aparecimento de lesões nos pés.

Local da atividade: Dia 09 de julho em Iati - PE na Unidade Básica de Saúde Santa Terezinha.

Público alvo: Indivíduos diabéticos e hipertensos, seus familiares e respectivos cuidadores; presentes onze pessoas.

Objetivo geral: Levar informações relevantes, que conscientizem a população sobre o autocuidado, prevenção e cuidados com o pé diabético.

\section{Objetivos específicos da atividade:}

- Apresentar a oficina de forma simples e direta na Unidade Básica de Saúde.

- Sensibilizar a população da importância em prevenir e cuidar do pé diabético corretamente. 
Competências a serem alcançadas: reconhecer a saúde como direito e condições dignas de vida e atuar de forma a garantir a integralidade da assistência, entendida como conjunto articulado e contínuo das ações e serviços preventivos e curativos, individuais e coletivos, exigidos para cada caso em todos os níveis de complexidade do sistema.

Facilitadores: Thaís Ferro e Núbia Ivo.

Data: $31 / 07 / 2019$

\section{Apresentação:}

Oficina realizada na UBS Santa Rosa com tema: prevenção do pé diabético com o grupo de idosos da unidade, abordando o autocuidado, a prevenção e os cuidados com o pé diabético.

Local da atividade: Dia 31 de julho em Santa Rosa no município de Iati - PE na Unidade Básica de Saúde Santa Rosa.

Público alvo: Indivíduos diabéticos e hipertensos, seus familiares e respectivos cuidadores; presentes onze pessoas.

Objetivo geral: Levar informações relevantes, que conscientizem a população sobre o autocuidado, prevenção e cuidados com o pé diabético.

\section{Objetivos específicos da atividade:}

- Apresentar a oficina de forma simples e direta na Unidade Básica de Saúde.

- Sensibilizar a população da importância em prevenir e cuidar do pé diabético corretamente.

Competências a serem alcançadas: reconhecer a saúde como direito e condições dignas de vida e atuar de forma a garantir a integralidade da assistência, entendida como conjunto articulado e contínuo das ações e serviços preventivos e curativos, individuais e coletivos, exigidos para cada caso em todos os níveis de complexidade do sistema.

Figura 1. Boneca terapêutica com premiação.

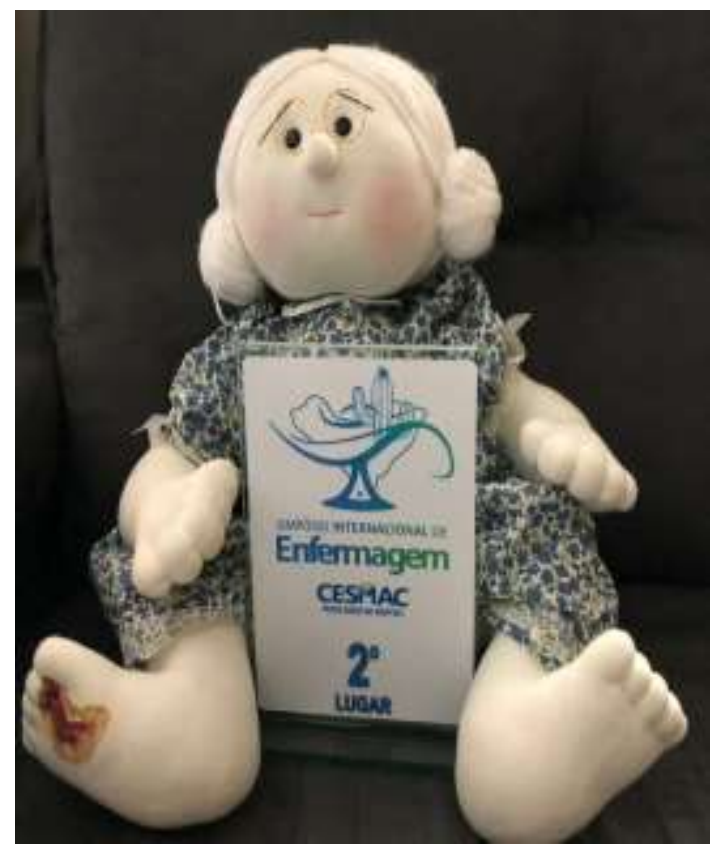

Fonte: Autores.
Figura 2. Boneca terapêutica com premiação e uma criadora.

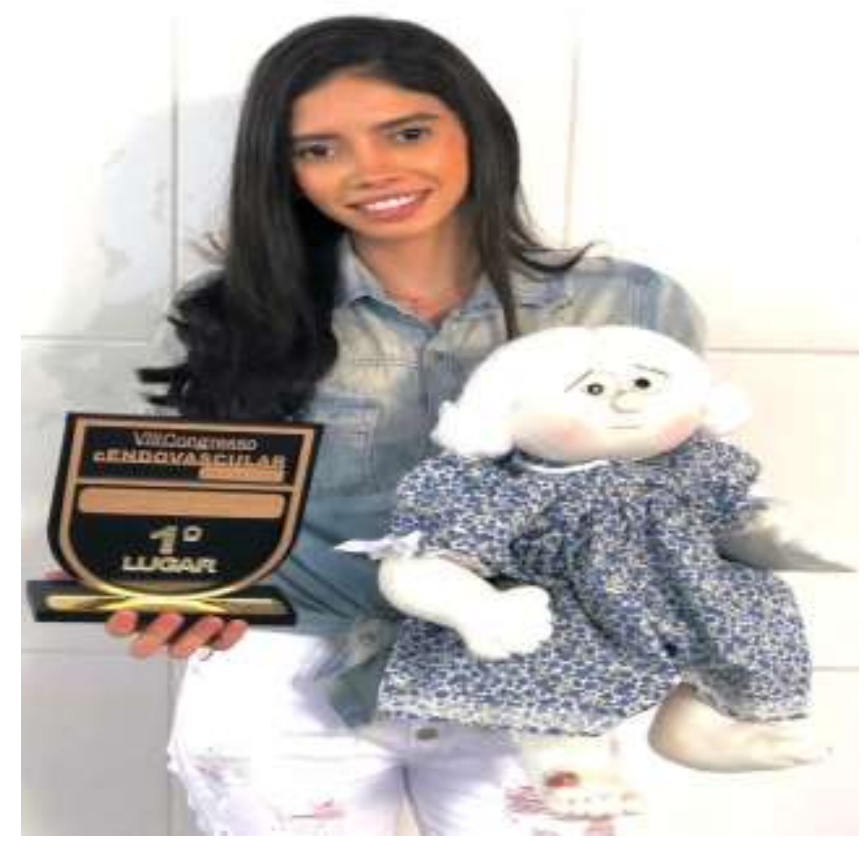

Fonte: Autores.

Boneca terapêutica Aurora (Figura 1), em premiação internacional no Simpósio Internacional de Enfermagem da Faculdade CESMAC do Sertão, Palmeira dos Índios - AL. Onde foi explanado o tema: Uso da metodologia da problematização 
com o arco de Maguerez na produção de uma boneca terapêutica como estrategia de educação em saúde para o público idoso, que foi premiado em $2^{\circ}$ lugar. Onde devemos nos atentar para o pé diabético em uma boneca terapêutica idosa.

Boneca terapêutica Aurora juntamente com um de seus inventores e autor principal do presente periódico (Figura 2), em premiação internacional no VIII Congresso Cendovascular Internacional - III Encontro Alagoano de Enfermagem nos Cuidados das Feridas.

\section{Discussão}

Para garantir que a intervenção fosse exitosa, o material didático foi cuidadosamente confeccionado pelos promotores da ação. Cada encontro demandou o material. O planejamento das atividades foi elaborado de maneira detalhada, conforme o preconizado por Cyrino (2016), que diz que o planejamento meticuloso das ações é uma forma de respeito ao grupo.

A ação educativa em saúde é um processo dinâmico que objetiva a capacitação dos indivíduos ou grupos em busca de melhoria das condições de vida. Destaca-se que nesse processo, os indivíduos podem ou não adotarem os novos comportamentos frente aos problemas de saúde. Não basta apenas o seguimento das normas estabelecidas pelos profissionais, e sim realizar a educação em saúde num processo que estimule o diálogo, a indagação, a reflexão, o questionamento e a ação partilhada.

Segundo Oliveira (2011) a questão do autocuidado é prejudicada ou insatisfatória em muitas situações, em especial, nos casos de pacientes portadores de DM.

Portanto, faz-se necessária uma abordagem multiprofissional, a fim de promover a propagação do conhecimento e empoderamento para o autocuidado. No decorrer dos encontros, os idosos participaram ativamente na realização de diversos questionamentos, em especial sobre o uso de plantas medicinais como método terapêutico para a DM. As dúvidas foram discutidas com intuito de aumentar a compreensão dos idosos sobre o assunto em questão. É fundamental ressaltar que o cuidado com os pés foi um dos maiores desafios encontrados. A maioria dos participantes presentes referiram não fazer a avaliação constante e não demonstraram preocupação com os pés.

A estratégia da educação em saúde é um processo dinâmico e o espaço do grupo representou um aprendizado significativo para todos os participantes. Os profissionais de saúde como mediadores da atividade foram capazes de estimular o diálogo, a indagação e a reflexão. Além disso, puderam rever e, também, reorientar suas práticas. Deste modo, os encontros em grupo são problematizados, refazendo modos de pensar e de agir, sendo um espaço de construção do saber e trocas efetivas.

Segundo Freire (2010), educação implica em uma intervenção no mundo, que se alicerça em uma prática mobilizadora, que exige compartilhamento do conhecimento cientifico e empírico. Esse processo deve ser preparado sem arrogância, pelo contrário, com humildade e o respeito jamais negado ao educando e ao seu saber de experiência, que deve ser buscado com ele. As oficinas permitiram aos facilitadores e aos idosos o reconhecimento de que a saúde na velhice não está relacionada somente ao bem-estar físico, mas ao contexto de inserção do idoso em relação ao lazer e a socialização, ou seja, um processo que percorre etapas que envolvem a família, a sociedade e o próprio indivíduo.

$\mathrm{Na}$ análise dos estudos, percebeu-se que há evidências na literatura científica de ações educativas que busquem a participação ativa dos idosos, todavia, ainda são consideradas inadequadas para provocar mudanças de comportamento nos sujeitos e inapropriadas por utilizarem metodologias que não condizem com os princípios da educação em saúde. Intervenções educativas são iniciativas que podem melhorar a qualidade de vida na terceira idade e, os profissionais de saúde devem promover a participação ativa dos idosos nas atividades, as quais devem ser baseadas nas necessidades elencadas pela população (Tavares, et al., 2012).

Ao propor o trabalho em grupo com idosos nas Unidades Básicas de Saúde, as diretrizes do Ministério da Saúde apontam igualmente que os coordenadores devem considerar as peculiaridades socioeconômicas dos participantes e os saberes disponíveis 
nas comunidades. Cabe lembrar que idosos têm longa experiência de vida e conhecimento de práticas tradicionais que devem ser trazidas ao debate sobre saúde.

Em se tratando de idosos, conceber a ação educativa em saúde como processo formativo do humano, transcende a abordagem de conteúdos específicos sobre a doença, prevenção e tratamento e possibilita que interações mais profundas e significativas se estabeleçam entre os sujeitos envolvidos, com riqueza de aprendizado para ambas as partes. Os grupos serão tão mais acolhedores aos idosos e valiosos em seu processo de cuidado e de vida quanto mais se constituírem como espaço de significação do envelhecer e do adoecer, ultrapassando o discurso técnico e possibilitando a expressão dos aspectos situacionais, emocionais e subjetivos que envolvem a vida dos sujeitos (Noelle, 2012).

É nessa linha que a expressão criativa e o lúdico integram várias experiências analisadas, sobretudo as orientadas pela educação permanente em saúde.

No trabalho de Santos (2011), o uso de técnicas dramatúrgicas permitiu a integração de conteúdos não só pela articulação dos saberes de várias áreas, mas por permitir o diálogo através de expressões verbais e corporais. Na experiência de Menezes (2011), estratégias metodológicas baseadas em conversa e alegria são privilegiadas na busca de gerar momentos de reflexão e transformação (Noelle, 2012).

Como afirma Dantas (2011), as várias linguagens da arte e das práticas populares permitem tocar em dimensões mais totalizadoras do sujeito, em uma obra que vincula desejo e cognição, intuição e sensibilidade e suscita a reflexão sobre a humanização nas práticas de saúde e o papel dos profissionais como educadores.

As oficinas educativas configuram uma importante estratégia de educação em saúde por se constituírem como espaços reais de expressão individual e coletiva de vivências e troca de saberes. A partir das atividades desenvolvidas com os idosos, foi possível desvelar verdades, verificar as fragilidades e orientá-los sobre o uso correto das medicações, possibilitando um espaço de interação positiva com os discentes e docentes de Enfermagem.

A enfermagem, ao trabalhar o estilo de vida saudável, deve ressaltar a importância do desenvolvimento do autocuidado durante todas as etapas do ciclo vital, objetivando garantir uma velhice saudável através das mudanças de hábito e adoção de novos padrões de comportamento (Menezes, 2011).

Promover a educação em saúde para idosos com a utilização de atividades lúdicas foi um desafio, portanto, os resultados foram considerados positivos, pois propiciaram uma ativa participação dos idosos, fazendo com que os mesmos se tornassem corresponsáveis pelo processo saúde-doença. Tais benefícios podem também ser observados em outros estudos que usaram a atividade lúdica com idosos, como Fleurí (2013), que em atividade lúdica realizada junto a idosos institucionalizados, constatou que elas foram de grande valia e trouxeram benefícios físicos e psicológicos para os idosos participantes da ação.

Assim como Andrade (2012), constatamos que o uso da atividade lúdica com idosos favorece a melhoria da qualidade de vida dessa população e que os estímulos por meio das brincadeiras visam diminuir as resistências ao tato, ao contato, ao movimento e dar mais sentido à vida, além de aumentar a possibilidade de adoção de hábitos saudáveis.

Por fim, fazemos coro com Fleurí (2013) e Meneses (2013), ao afirmarem que a realização de atividades lúdicas com idosos é um importante aliado para o fortalecimento da autonomia destes indivíduos.

\section{Conclusão}

Constatou-se que a boneca terapêutica criada, Aurora, é uma estratégia educativa relevante, direcionada para a conscientização da necessidade de cuidados diários adequados com os pés, trazendo resultados positivos para prevenção de complicações no pé diabético. Dessa forma, evidencia que através da criatividade podemos melhorar e transformar a condição do processo de aprendizado em algo lúdico e efetivo. 
Cabe destacar ainda, que o brinquedo terapêutico da mesma forma que é eficaz para abordar temas com crianças como hospitalização, preparação para cirurgias e punção venosa, permitindo ela enfrentar e compreender situações atípicas para idade, também oferece resultados efetivos na abordagem de temas de educação em saúde para os idosos como administração de insulina, vacinas, alzheimer, câncer, prevenção de quedas e diversos outros. Assim sendo, a aplicação do brinquedo terapêutico pode ser direcionada a outros públicos, não apenas às crianças.

Evidencia-se, nesse projeto, que a boneca terapêutica favoreceu a comunicação com a população idosa e com os seus cuidadores, aliada ao uso de uma linguagem simples e acessível sobre os cuidados para prevenção do pé diabético. Vale salientar que a empatia e o carinho que uma boneca idosa foi capaz de gerar, principalmente, no público mais idoso, despertando o interesse para o que estava sendo transmitido nas oficinas, promovendo a interação e favorecendo o diálogo.

Este projeto revelou que os diabéticos e os cuidadores muitas vezes não têm consciência da importância de observar os pés diariamente, muitos desconhecem as complicações que podem surgir na ausência de um cuidado básico, que na maioria dos casos pode ser realizado pelos mesmos. Atitudes simples, baratas e passíveis de implementar diariamente através da simulação destes cuidados na boneca. O autocuidado dos diabéticos com os pés é tão importante quanto o controle glicêmico.

Dada a grandeza do problema relacionado à complexidade do pé diabético e sua alta prevalência, torna-se necessário para sua prevenção e controle, o desenvolvimento de ações para que o sujeito construa o seu conhecimento e não seja um mero expectador.

A realização das intervenções educativas em uma abordagem grupal evidenciou melhoria nos aspectos da alimentação saudável e do cuidado com os pés, antes não promovidas conforme verificado no grupo-controle. Ressalta-se que para manter esse resultado satisfatório, bem como ampliar o autocuidado, essa prática deve ser contínua, na qual a realização da intervenção poderá interferir diretamente nos aspectos clínicos.

Há diversas vantagens neste tipo de estudo, como o envolvimento dos idosos, a aprendizagem coletiva para o cuidado com os pés, além de os idosos multiplicarem informações, pois eles relataram, durante as intervenções, que orientariam outros idosos e familiares com DM que não estavam presentes quanto ao conteúdo abordado durante a atividade educativa.

Verificou-se, ao longo do desenvolvimento das atividades educativas, que houve uma contribuição significativa para a conscientização dos idosos quanto à relevância do seu engajamento no processo de autocuidado, que leva à autonomia e consequente ao empoderamento, com melhoria da qualidade de vida, além da consolidação de laços sociais e afetivos entre os participantes

Cabe mencionar a importância do desenvolvimento de atividades grupais pelos enfermeiros, voltadas para os idosos na atenção primária, principalmente para a prevenção de incapacidades e complicações relacionadas às doenças crônicas, em particular, o DM.

Com esse intuito, faz-se necessário expandir essa discussão fomentando com outras pesquisas sobre esta mesma temática, para que se possa mensurar o nível de esclarecimento da população sobre a neuropatia diabética. Por isso, estudos futuros precisam ser realizados, com intervenções padronizadas e maior quantitativo de participantes a longo prazo, principalmente, intervenções focadas na realidade dos sistemas de saúde do Brasil.

\section{Agradecimentos}

Gostaria de agradecer a Deus primeiramente e a todos os profissionais que colaboraram de forma positiva no desenvolvimento desta pesquisa; aos meus colegas de pesquisa e a população de Iati-PE; Palmeira dos Índios-AL e ArapiracaAL que se fizeram presente em todas as ações extensionistas. 
Research, Society and Development, v. 10, n. 4, e22410413992, 2021

(CC BY 4.0) | ISSN 2525-3409 | DOI: http://dx.doi.org/10.33448/rsd-v10i4.13992

\section{Referências}

Augusto, C. A., et. al. (2013). Pesquisa Qualitativa: rigor metodológico no tratamento da teoria dos custos de transação em artigos apresentados nos congress os da Sober (2007-2011). Rev. Econ. Sociol. Rural, 51(4), 745-764.

Boell, J., Ribeiro, R. \& Silva, D. M. (2014). Fatores de risco para o desencadeamento do pé diabético. Revista Eletrônica De Enfermagem, 16(2), 386-93.

Cardoso, N. A., et al. (2018). Fatores de risco para mortalidade em pacientes submetidos a amputações maiores por pé diabético infectado. J. vasc. bras., 17(4), 296-302.

Carvalho, R. P., Carvalho, C. P. \& Martins, D. A. (2010). Aplicação dos Cuidados com os Pés entre Portadores de Diabetes Mellitus. Cogitare Enfermagem, $15(1)$.

Cecilio, H. P. N., et al. (2015). Comportamentos e comorbidades associados às complicações microvasculares do diabetes. Acta Paulista de Enfermagem, 28(2), 113-119.

Charmaz, K. A. (2009). Construção da teoria fundamentada: guia prático para análise qualitativa. Artmed

Cyrino, R., et al. (2016). Atividades lúdicas como estratégia de educação em saúde com idosos. Revista Ciência em Extensão, 12 (3), $154-163$.

Dantas, V. (2011). Acolhimento e Educação Popular: pra onde aponta essa trilha. 24.

Fleurí, A. C. P., et al. (2013). Atividades lúdicas com idosos institucionalizados. Enfermagem Revista, 16(1), 50-57.

Freire, P. (2010). Pedagogia da autonomia: saberes necessários à prática educativa. Paz e Terra.

Freitas, T. P. P., et al. (2016). Contribuições da extensão universitária na formação de acadêmicos de enfermagem. Revista de Enfermagem da UFSM, [S.1.], $6(3), 307-316$.

Galdino, Y. L. S., et al. (2019). Validation of a booklet on self-care with the diabetic foot. Revista Brasileira de Enfermagem, $72(3), 780-787$.

Gomes, D. M., et al. (2017). Ressignificação do Cuidado de uma Pessoa com Diabetes e Pé Diabético: Relato de Experiência. Revista de Enfermagem do CentroOeste Mineiro $; 1509$.

Glaser, B. G. \& Strauss, A. (1967). The discovery of grounded theory: strategies for qualitative research. Aldine.

Grittem, L., Meier, M. J. \& Zagonel, I. P. S. (2008). Pesquisa-ação: uma alternativa metodológica para pesquisa em enfermagem. Texto contexto - enferm., Florianópolis, 17(4), 765-770.

Lucoveis, M. L. S., et al. (2018). Grau de risco para úlcera nos pés por diabetes: avaliação de enfermagem. Rev. Bras. Enferm. 71(6), 30413047 .

Macedo, M. M. L., et al. (2017). Adherence to self-care practices and empowerment of people with diabetes mellitus: a randomized clinical trial. Rev. Esc. Enferm. USP.

Meneses, D. L. P., et al. (2013). Dupla face da velhice: o olhar de idosos sobre o processo de envelhecimento. Enfermagem em Foco, 4(1), 15-18.

Menezes, R. L., et al. (2011). Estudo longitudinal dos aspectos multidimensionais da saúde de idosos institucionalizados. Rev Bras GeriatrGerontol, 14(3):48596.

Minayo, M. C. S. (2012). Análise qualitativa: teoria, passos e fidedignidade. Ciênc. saúde coletiva, 17(3), 621-626.

Noelle, W. (2012). Educação popular para promoção da saúde e empoderamento da comunidade: uma revisão da literatura. Health Promotion Internacional. 27(3), 356- 371.

Pessoa, V. M., et al. (2013). Pesquisa-ação: proposição metodológica para o planejamento das ações nos serviços de atenção primária no contexto da saúde ambiental e da saúde do trabalhador. Interface (Botucatu), 17(45), 301-314.

Pereira, C. N. M. S. C., et. al. (2012). The effect of educational intervention on the disease knowledge of diabetes mellitus patients. Rev Latino Am Enfermagem. 20(3):478-85

Rodrigues, H. F., et. al. (2014). Cuidado de los pies: conocimiento de los individuos con diabetes mellitus. Enferm. glob, 13(35), 41-51.

Roediger, M. A., et al. (2018). Diabetes mellitus referida: incidência e determinantes, em coorte de idosos do município de São Paulo, Estudo SABE - Saúde, Bem-Estar e Envelhecimento. Ciênc.saúde coletiva, 23(11), 3913-3922.

Santos, M. V. (2011). O processo participativo de idosos através de experiências e práticas do movimento de educadores populares. Rev APS, 14(4):378-88.

Scain, S. F., Franzen, E., \& Hirakata, V. N. (2018). Riscos associados à mortalidade em pacientes atendidos em um programa de prevenção do pé diabético. Rev. Gaúcha Enferm., 39, e20170230.

Silva, A. F. L., Ribeiro, C. D. M. \& Silva Júnior, A. G. (2013). Thinking of university extension as a health education field: an experience at the Fluminense Federal University, Brazil. Interface (Botucatu), 17(45), 371-84.

Silva, L. W. S., et al. (2016). Promoção da saúde de pessoas com diabetes mellitus no cuidado educativo preventivo do pé-diabético. Ciencia y Enfermaria, 103116. 
Research, Society and Development, v. 10, n. 4, e22410413992, 2021

(CC BY 4.0) | ISSN 2525-3409 | DOI: http://dx.doi.org/10.33448/rsd-v10i4.13992

Silva, S. O., et al. (2018). Consulta de enfermagem para pessoas com diabetes mellitus: experiência com metodologia ativa. Rev. Bras. Enferm. Brasília, 71(6), 3103-3108.

Tavares, D. M. S., Dias, F. A., \& Munari, D. B. (2012). Qualidade de vida de idosos e participação em atividades educativas grupais. Acta Paul Enferm.u 25(4): 601-606. 